“(C) 2015 IEEE. Personal use of this material is permitted. Permission from IEEE must be obtained for all other uses, in any current or future media, including reprinting/republishing this material for advertising or promotional purposes, creating new collective works, for resale or redistribution to servers or lists, or reuse of any copyrighted component of this work in other works." 


\title{
A closed-form expression for coverage probability of random cellular network in composite Rayleigh-Lognormal fading channels
}

\author{
Sinh Cong Lam, Roshanak Heidary, Kumbesan Sandrasegaran \\ Centre for Real-Time Information Networks, Faculty of Engineering and Information Technology, \\ University of Technology, Sydney, Australia
}

\begin{abstract}
Poisson point process (PPP) network model in which base stations (BSs) and users have Poisson distributions has been recently used to replace grid model for analyzing the performance of cellular networks. The closed-form for the coverage probability of a typical user that connects to the closest base station (BS), however, is only found in case of high transmission signal-tonoise (SNR) and only in Rayleigh fading. This paper derives a closed-form expression for the network coverage probability in composite Rayleigh-Lognormal for both low and high SNR. The analytical results show that the coverage probability is proportional to path loss exponent coefficient, $\alpha$, and inversely proportional to exponential function of $\frac{1}{S N R}$. The analytical results are also verified by Monte Carlo simulations.
\end{abstract}

Keywords-Coverage Probability, Ergodic capacity, random cellular network, Rayleigh-Lognormal, Gauss-Hermite quadrature, Gauss- Legendre quadrature.

\section{INTRODUCTION}

In realistic mobile radio scenarios in urban areas, the multipath effect at the mobile receiver due to scattering from local scatters such as buildings in the neighborhood of the receiver causes a fast fading while the variation in the terrain configuration between the base-station and the mobile receiver causes a slow shadowing. Therefore, the mobile radio signal envelope is usually composed of a small scale multipath fading component superimposed on a larger scale or slower shadowing component. It is well known that the signal envelope of the multipath component can be modeled as a Rayleigh distributed random variable (RV), and its power can be modeled as an exponential RV. Thus the path power gain has a mixed Rayleigh-Lognormal distribution which is also known as the Suzuki fading distribution model [1].

The traditional grid network model with deterministic BS locations is no longer accurate to estimate the performance of multi-cell wireless networks [2]. Poisson point process (PPP) network model [2], in which the BS locations are modeled as a homogeneous spatial Poisson point process, has been developed as a more accurate and flexible tractable model for cellular network. As in well-known network models, a typical user is allowed to associate with the closest or the strongest BS. In strongest model, a user measures Signal-to-Interferenceplus-Noise ratio (SINR) from several BSs and selects the BS with the highest SINR while in closest model, the distances between a user and BSs are estimated, and the BS, which is nearest to the user, is selected.
Most recent works on PPP model focused on deriving exact formulas for performance of a typical user in only Rayleigh based on coverage probability approach[2]. Due to the complexity of mathematical manipulations, the mathematical expression stopped at two layer integrals for closest model [2, $3]$ and one layer integrals for strongest model [4, 5]. In these frameworks, the closed-form expressions were only found in case of ignoring Gaussian noise.

Authors in [6] proposed a new method to calculate average capacity in generalized fading channels based on moment generating function (MGF) which could avoid the complexity of coverage probability approach. However, the final equations were not exactly simple because they contained the Gauss hypergeometric function [7] which is expressed as an integral. Another work, that evaluated the effect of Rayleigh and shadowing, was considered in [8] where shadowing was not incorporated in channel gain and can be constant when the origin PPP model is rescaled. Instead of rescaling network model, authors in [9] introduced a new approach to derive the coverage probability mathematical expression. However, this approach still stopped at the two layer integrals and is only used for zero noise networks (neglecting noise).

This paper is based on coverage probability frameworks and uses the exponential form of probability density function (PDF) of composite Rayleigh-Lognormal random variable (RV) and Gauss-Legendre to obtain the closed-form expression for coverage probability of a typical user in the closest PPP network model.

\section{SYSTEM MODEL}

\section{A. Network Topology}

We consider a PPP cellular network (as shown in Figure 1) in which the locations of BSs are distributed as a homogeneous spatial Poisson point process (PPP) with density $\lambda$. A typical user is randomly located according to an independent stationary point process in a Voronoi cell and has connection with the closest BS [2].

Without loss of generality, a typical user is assumed to be located at the origin and served by a BS at distance $r$. The probability density function (PDF) of $r$ is given by:

$$
f_{r}(r)=2 \pi \lambda e^{-\pi \lambda r^{2}}
$$




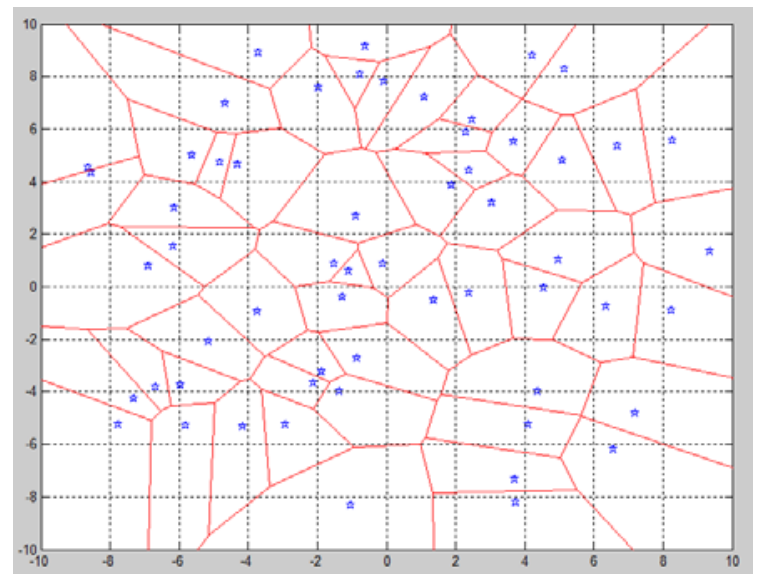

Figure 1: An example of PPP network model with $\lambda=0.2$

In our analysis, we assume that all BSs transmit continuously in all time slots and at constant power, although if their operation is interrupted with coefficient $\beta$, then the density of BSs should be changed from $\lambda$ to $(1-\beta) \lambda$ [5].

\section{B. Channel model}

In downlink cellular network, the signal transmitted over distance $r$ suffers free-space path loss, fast fading as well as slow fading.

The free-space path loss (FSPL) can be defined as the loss of signal strength of an electromagnetic signal originated from the line of sight path through free space without any obstacles that cause any reflection or diffraction. For a receiver at distance $r$ from the transmitter, the received power is $P_{r}=P^{-\alpha}$ in which $\alpha$ is path-loss coefficient.

The probability density function (PDF) of power gain $g$ of signal experiencing Rayleigh and Lognormal fading is found from the probability density function (PDF) of the product two cascade channels [1].

$$
=\int_{0}^{f_{R-\operatorname{Ln}}(g)} \frac{1}{x} \exp \left(-\frac{g}{x}\right) \frac{1}{x \sigma_{z} \sqrt{2 \pi}} \exp \left(-\frac{\left(10 \log _{10} x-\mu_{z}\right)^{2}}{2 \sigma_{z}^{2}}\right) d x
$$

Then, its cumulative density function is given by: (see Appendix A)

$$
F_{R-L n}(g)=\sum_{n=1}^{N_{p}} \frac{w_{n}}{\sqrt{\pi}}\left[1-\exp \left(-\frac{g}{\gamma\left(a_{n}\right)}\right)\right]
$$

in which

- $w_{n}$ and $a_{n}$ are, respectively, the weights and the abscissas of the Gauss-Hermite polynomial. The approximation becomes more accurate with increasing approximation order $N_{p}$. For sufficient approximation, we use $N_{p}=12$.

- $\gamma\left(a_{n}\right)=10^{\left(\sqrt{2} \sigma_{z} a_{n}+\mu_{z}\right) / 10} ; \mu_{z}$ and $\sigma_{z}$ are mean and variance of Rayleigh-Lognormal random variable.

The associated signal power which a typical user receives from its BS at distance $r$ in composite Rayleigh-Lognormal fading is:

$$
S=P g r^{-\alpha}
$$

\section{Signal-to-interference-noise ratio (SINR)}

We denote the set of interfering BS as $\theta ; r_{u}$ and $g_{u}$ are the distance and channel gain from a typical user to interference $\mathrm{BS}$ respectively. Since a user connect to the closest BS, $r_{u}>$ $r$. The intercell interference that causes a user is obtained by

$$
I_{\theta}=\sum_{\theta} P g_{u} r_{u}^{-\alpha}
$$

Combining (4) and (5), the received instantaneous SINR at a typical user is found from Equation (6)

$$
S I N R=\frac{P g r^{-\alpha}}{\sigma^{2}+I_{\theta}}
$$

\section{COVERAGE PROBABILITY}

The coverage probability $P_{c}$ of a typical user for a given threshold $T$ is as the probability of event in which the SINR is larger than a threshold. [2] proved that $P_{c}$ is a function of SINR threshold $T$, BS density $\lambda$ and attenuation coefficient $\alpha$. Then $P_{c}$ can be written as below:

$$
P_{c}(T, \lambda, \alpha \mid r)=\mathbb{P}(S I N R>T)
$$

This coverage probability is exactly the complementary part of the CDF of SINR, since the CDF is defined as $F_{c}(T, \lambda, \alpha \mid r)=$ $\mathbb{P}(\operatorname{SINR}<T)$.

Theorem 1: The coverage probability of a typical user in Rayleigh-Lognormal fading is

$$
P_{c}(T, \lambda, \alpha \mid r)=\sum_{n=1}^{N_{p}} \frac{w_{n}}{\sqrt{\pi}} \mathrm{e}^{-\frac{T}{\gamma\left(a_{n}\right) S N R} r^{\alpha}} \mathrm{e}^{-\pi \lambda r^{2} f_{S F R}(T, n)}
$$

where $S N R=\frac{P}{\sigma^{2}}$ is the signal-to-noise ratio at the transmitter, $C=T \frac{\gamma\left(a_{n_{1}}\right)}{\gamma\left(a_{n}\right)}$,

$f_{S F R}(T, n)=$

$$
\sum_{n_{1}=1}^{N_{p}} \frac{w_{n_{1}}}{\sqrt{\pi}}\left(\frac{1}{\alpha} C^{\frac{1}{\alpha}} \frac{\pi}{\sin \left(\frac{\pi(\alpha-1)}{\alpha}\right)}-\sum_{n_{G L}=1}^{N_{G L}} \frac{c_{i}}{2} \frac{C}{C+\left(\frac{x_{i}+1}{2}\right)^{\alpha}}\right)
$$

where $c_{i}$ and $x_{i}$ are weights and nodes of Gauss-Legendre rule respectively with order $N_{G L}$. In this paper, $N_{G L}=10$ is sufficient for accurate computation.

\section{Proof: See Appendix B}

It is observed from Theorem 1 that the coverage probability of a typical user is inversely proportional to exponential function of $\frac{1}{S N R}$ and $r^{\alpha}$ for cellular network with $\sigma^{2}>0$. In case of negligible noise $\sigma^{2}=0$, the coverage probability does not depend on the BS transmission power since $\frac{1}{S N R}=0$.

Lemma 1: The average coverage probability of a typical user over network in composite Rayleigh-Lognormal fading is

$P_{c}(T, \lambda, \alpha)=$ 


$$
4 \pi \lambda \sum_{i=1}^{N_{G L}} \frac{c_{i}\left(x_{i}+1\right)}{\left(1-x_{i}\right)^{3}} e^{-\pi \lambda\left(\frac{x_{i}+1}{1-x_{i}}\right)^{2}} P_{c}\left(T, \lambda, \alpha \mid r=\frac{x_{i}+1}{1-x_{i}}\right)
$$

Proof: The average coverage probability is achieved by integrating $P_{c}(T, \lambda, \alpha \mid r)$ in Equation (9) with variable $r>0$.

$$
P_{c}(T, \lambda, \alpha)=\int_{0}^{\infty} 2 \pi \lambda r e^{-\pi \lambda r^{2}} P_{c}(T, \lambda, \alpha \mid r) d r
$$

Let $r=\frac{t}{1-t}(0<\mathrm{t}<1)$. By using Gauss-Legendre rule, we can obtain the desired result.

This closed-form expression for coverage probability of a typical user in Rayleigh-Lognormal fading is much more simple and general expression than the previous results in $[8$, 9]. This result does not only indicate the dependence of network coverage on distance between the transmitter and receiver, but also shows the impact of transmission SNR on cell expansion.

$$
\begin{aligned}
\text { For } \sigma^{2} & =0 \text {, Equation (10) becomes: } \\
P_{c}(T, \lambda, \alpha) & =\sum_{n=1}^{N_{p}} \frac{w_{n}}{\sqrt{\pi}} \frac{1}{1+f_{S F R}(T, n)}
\end{aligned}
$$

It is observed that in this case, the coverage probability does not depend on the density of the BS. This means the coverage probability is consistent with the changes of number of BS[2].

Lemma 2: The coverage probability of a typical user over network in Rayleigh fading only.

$$
P_{c}(T, \lambda, \alpha \mid r)=\mathrm{e}^{-T \frac{1}{S N R} r^{\alpha}} \mathrm{e}^{-\lambda r f_{S F R}(T)}
$$

where

$$
f_{S F R}(T)=\frac{\pi \frac{1}{\alpha} T^{\frac{1}{\alpha}}}{\sin \left(\frac{\pi(\alpha-1)}{\alpha}\right)}-\sum_{n_{G L}=1}^{N_{G L}} \frac{c_{i}}{2} \frac{T}{T+\left(\frac{x_{i}+1}{2}\right)^{\alpha}}
$$

Proof: Rayleigh fading is a special case of composite Rayleigh-Lognormal fading with $\sigma_{z}=0$ and given that $\sum_{n_{1}=1}^{N_{p}} \frac{w_{n}}{\sqrt{\pi}}=1$, then the coverage probability in this case is derived by Equation (13) and (14).

The average coverage probability over network is calculated by integrating Equation (13) with variable $r>0$, and then its closed-form is expressed as Equation (10) where $P_{c}(T, \lambda, \alpha \mid r)$ is defined in Equation (13). This analytical result is comparable to the corresponding result for Rayleigh fading given in [2].

\section{ERGODIC CAPACITY}

Theorem 2: The average ergodic capacity of a typical user that connects to the closest BS is given by:

$$
\mathbb{C}(\lambda, \alpha)=\sum_{i=1}^{N_{G L}} \frac{2 c_{1 i}}{\left(1-x_{1 i}\right)^{2}} P_{c}\left(t=\exp \left(\frac{x_{1 i}+1}{1-x_{1 i}}\right)-1, \lambda, \alpha\right)
$$

where $c_{1 \mathrm{i}}$ and $\mathrm{x}_{1 \mathrm{i}}$ are weights and nodes of Gauss-Legendre rule with order $N_{G L} ; P_{c}$ as defined in Equation (10).

Proof: The ergodic capacity is defined by Shannon formula

$$
\mathbb{C}(\lambda, \alpha)=\mathbb{E}[\ln (1+\operatorname{SINR})]
$$

in which the average is evaluated over entire PPP network and fading distribution. Then the CDF function of average rate is:

$$
\begin{aligned}
F_{c}(\lambda, \alpha) & =\int_{0}^{\infty} \mathbb{P}[\ln (1+\operatorname{SINR})>t] d t \\
& =\int_{0}^{\infty} P_{c}\left(T=e^{t}-1, \lambda, \alpha\right) d t
\end{aligned}
$$

Processing the same way as in Equation (11), the average probability is achieved as in Equation (15).

\section{SimULATION AND DISCUSSION}

In this section, we use numerical method and Monte Carlo simulations to validate the theoretical analysis and to visualize the relationship between coverage probability and related parameters. In Figure 2, 3 and 4, the solid lines which present the theoretical analysis visually match with the dotted lines which present the simulation results. These confirm the accuracy of theoretical analysis results.

With higher $\alpha$, total power of interfering signals sees a faster decrease rate over distance than desired signal since the user receives only one useful beam from serving cell and often suffers more than one interfering beams. The coverage probability is, hence, inversely proportional to FSPL exponential coefficient $\alpha$. Figure 2 indicates that in the case of SINR threshold is $0 \mathrm{~dB}$ and SNR $=10 \mathrm{~dB}$, when $\alpha$ increases from 3.0 to 3.2 and ends at 3.5 , the coverage probability will increase by $16.20 \%$ and $30.3 \%$.

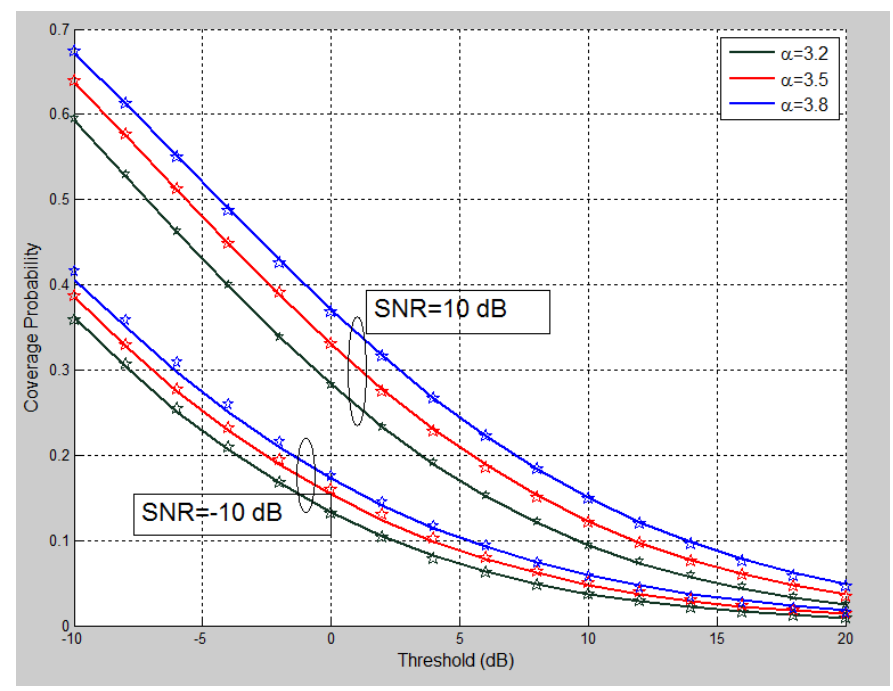

Figure 2: Variation of coverage probability with threshold for $\mu_{z}=-7.3683 \mathrm{~dB}, \sigma_{z}=8 \mathrm{~dB}$ and three values of attenuation coefficient $\alpha$.

It is observed from Figure 3 that in sparse networks (small $\lambda$ ), an increase in transmission power can significantly increase the coverage probability while in dense network 
(large $\lambda$ ), the transmission power only has a slight effect on coverage probability.

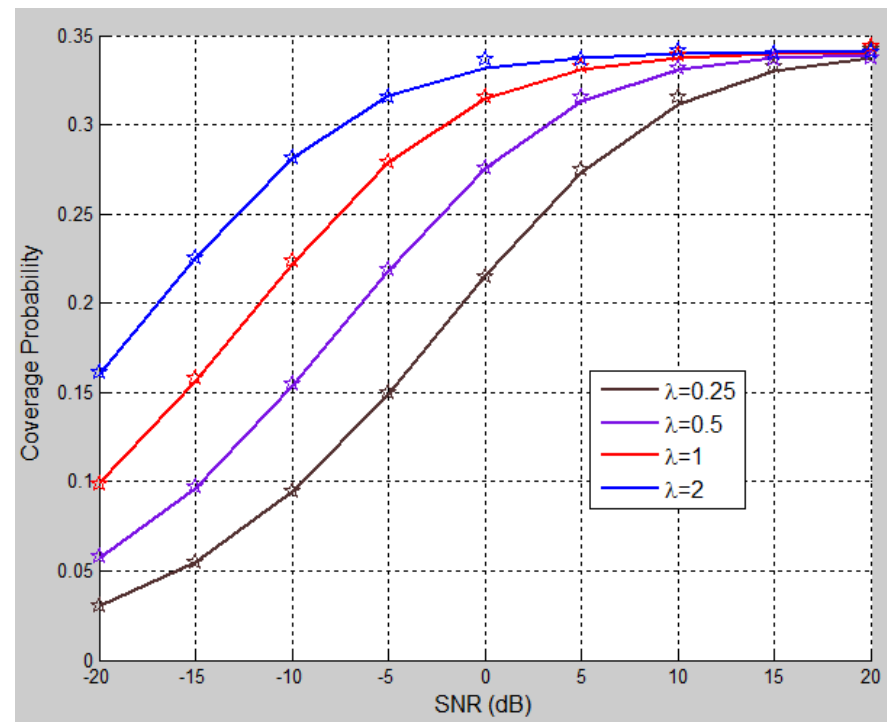

Figure 3: Variation of coverage probability with transmission SNR for $\mu_{z}=-7.3683 d B, \sigma_{z}=8 d B$, SINR threshold $T=0 d B$ and for many values of BS density

However, for both sparse and dense networks, the coverage probability reaches the upper bound when SNR is high (SNR > 20dB) because increasing of transmission power is counter-balanced by increasing interference.

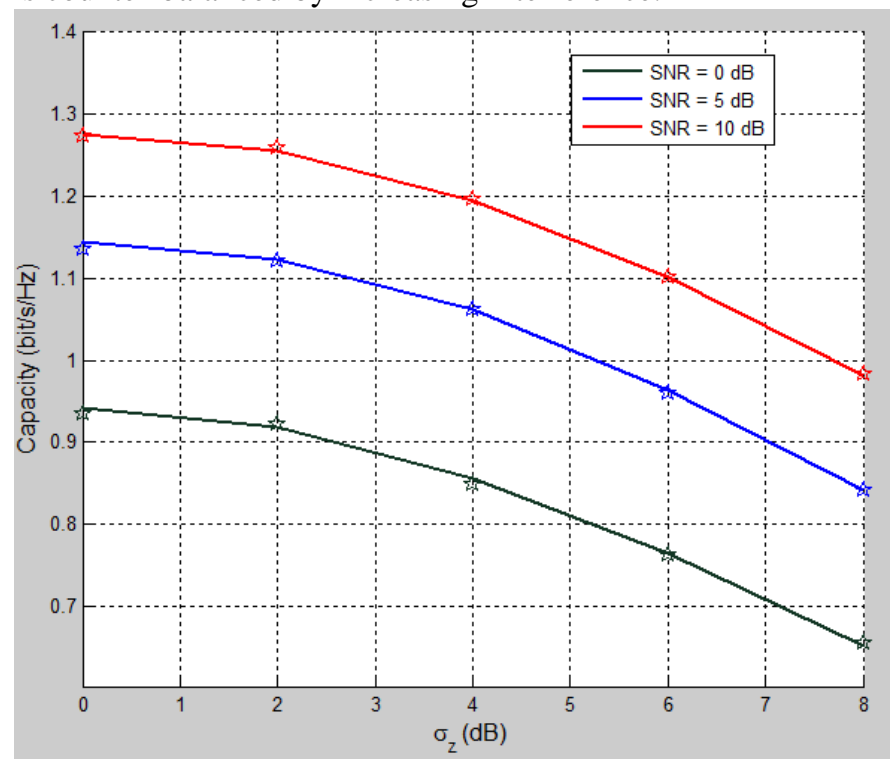

Figure 4: Variation of average capacity with SNR and $\sigma_{z}$

From Figure 4, we can see the average capacity is inversely proportional with shadowing standard deviation $\sigma_{z}$. However, it is interesting that the difference between average capacities with three values of SNR does not depend on $\sigma_{z}$. For instant, the gap between average capacity achieving when $\mathrm{SNR}=0$ and $5 \mathrm{~dB}$ is a constant and equals 0.1370 .

\section{CONCLUSION}

This paper presents a closed-form expression for coverage probability of a typical user associated with the closest BS in random cellular network in composite Rayleigh-Lognormal fading. The exact mathematical analysis for both low SNR and high SNR based on two well-known approximation rules, called Gauss-Hermite and Gauss- Legendre quadrature shows that the average coverage probability is inversely proportional to exponential function of $\frac{1}{S N R}$ for low SNR , and only depends on SINR threshold and path loss attenuation coefficient in high SNR. These analytical results are also expandable for complex PPP networks which enable frequency reuse and with $K(K>1)$ tier.

\section{APPENDIX A}

The probability density function (PDF) of power gain $g$ in Rayleigh-Lognormal fading is shown in Equation (2)

Let $t=\frac{10 \log _{10} g-\mu_{z}}{\sqrt{2} \sigma_{z}}=>g=10^{\left(\sqrt{2} \sigma_{z} t+\mu_{z}\right) / 10}=\gamma(t)$

Then

$$
f_{R-L n}(g)=\int_{0}^{\infty} \frac{1}{\sqrt{\pi}} \frac{1}{\gamma(t)} \exp \left(-\frac{g}{\gamma(t)}\right) \exp \left(-t^{2}\right) d x
$$

By using Gauss-Hermite expansion [7], the PDF equals

$$
f_{R-L n}(g)=\sum_{n=1}^{N_{p}} \frac{w_{n}}{\sqrt{\pi}} \frac{1}{\gamma\left(a_{n}\right)} \exp \left(-\frac{g}{\gamma\left(a_{n}\right)}\right)
$$

Hence, the CDF of Rayleigh-Lognormal RV is

$$
F_{R-L n}(g)=\sum_{n=1}^{N_{p}} \frac{w_{n}}{\sqrt{\pi}}\left[1-\exp \left(-\frac{g}{\gamma\left(a_{n}\right)}\right)\right]
$$

\section{APPENDIX B}

$$
\begin{array}{r}
\mathbb{P}(\operatorname{SINR}>T)=\mathbb{P}\left(\frac{P g r^{-\alpha}}{I+\sigma^{2}}>T\right) \\
=\mathbb{E}\left(\sum_{n=1}^{N_{p}} \frac{w_{n}}{\sqrt{\pi}} \exp \left(-\frac{\operatorname{Tr}^{\alpha}\left(I+\sigma^{2}\right)}{P \gamma\left(a_{n}\right)}\right)\right)
\end{array}
$$

$$
\begin{aligned}
& =\sum_{\substack{n=1 \\
N_{p}}}^{N_{p}} \frac{w_{n}}{\sqrt{\pi}} \mathbb{E}\left[\exp \left(-\frac{\operatorname{Tr}^{\alpha}\left(I+\sigma^{2}\right)}{\operatorname{P\gamma }\left(a_{n}\right)}\right)\right] \\
& =\sum_{n=1}^{N_{p}} \frac{w_{n}}{\sqrt{\pi}} \exp \left(-\frac{\operatorname{Tr}^{\alpha} \sigma^{2}}{\operatorname{P\gamma }\left(a_{n}\right)}\right) \mathbb{E}\left(\exp \left(-\frac{\operatorname{Tr}^{\alpha} I}{\operatorname{P\gamma }\left(a_{n}\right)}\right)\right) \\
& \text { Let } \frac{\operatorname{Tr}^{\alpha}}{P \gamma\left(a_{n}\right)}=f(n) \text { then } \\
& =\sum_{n=1}^{N_{p}} \frac{w_{n}}{\sqrt{\pi}} \exp \left(-f(n) \sigma^{2}\right) \mathbb{E}(\exp (-f(n) I))
\end{aligned}
$$


Considering the expectation

$$
\begin{aligned}
\mathbb{E}(\exp (- & f(n) I))=\mathbb{E}\left[\exp \left(-f(n) \sum_{u \in \theta} P g_{u} r_{u}^{-\alpha}\right)\right] \\
& =\mathbb{E}_{\theta}\left[\mathbb{E}_{g_{u}} \prod_{u \in \theta} \exp \left(-f(n) P g_{u} r_{u}^{-\alpha}\right)\right] \\
& =\mathbb{E}_{\theta}\left[\prod_{u \in \theta} \mathbb{E}_{g_{u}}\left(\exp \left(-f(n) P g_{u} r_{u}^{-\alpha}\right)\right)\right]
\end{aligned}
$$

Since $g_{u}$ is Rayleigh-Lognormal fading channel then

$$
=\mathbb{E}_{\theta}\left[\prod_{u \in \theta} \sum_{n_{1}=1}^{N_{p}} \frac{w_{n_{1}}}{\sqrt{\pi}} \frac{1}{1+\gamma\left(a_{n_{1}}\right) f(n) P r_{u}^{-\alpha}}\right]
$$

Using the properties of PPP probability generating function [10]

$$
=\exp \left(-\pi \lambda\left(\sum_{n_{1}=1}^{N_{p}} \frac{w_{n_{1}}}{\sqrt{\pi}} \int_{r}^{\infty} 2\left[1-\frac{1}{1+\gamma\left(a_{n_{1}}\right) f(n) P r_{u}^{-\alpha}}\right]\right) r_{u} d r_{u}\right)
$$

Given that $\frac{T r^{\alpha}}{P \gamma\left(a_{n}\right)}=f(n)$ and letting $\left(\frac{r_{u}}{r}\right)^{2}=t$ then the integral becomes

$=r^{2} \int_{1}^{\infty}\left(1-\frac{1}{1+\gamma\left(a_{n_{1}}\right) f(n) P t^{-\alpha / 2}}\right) d t$

$=r^{2}\left[\int_{0}^{\infty}\left(\frac{C t^{-\alpha / 2}}{1+C t^{-\alpha / 2}}\right) d t-\int_{0}^{1}\left(\frac{C}{t^{\alpha / 2}+C}\right) d t\right]$

$$
=r^{2}\left[I_{1}-I_{2}\right]
$$

in which $C=T \frac{\gamma\left(a_{n_{1}}\right)}{\gamma\left(a_{n}\right)}$

$$
\text { - } I_{1}=\int_{0}^{\infty}\left(\frac{C}{t^{\alpha / 2}+C}\right) d t
$$

Using properties of Gama function [7], we obtain

$$
I_{1}=\frac{2}{\alpha} C^{\frac{2}{\alpha}} \frac{\pi}{\sin \left(\frac{\pi(\alpha-2)}{\alpha}\right)}
$$

- $\quad I_{2}=\int_{0}^{1}\left(\frac{C}{t^{\alpha / 2}+C}\right) d t$ can be approximated by using Gauss-Legendre rule [7]

$$
I_{2}=\sum_{n_{G L}=1}^{N_{G L}} \frac{c_{i}}{2} \frac{C}{C+\left(\frac{x_{i}+1}{2}\right)^{\alpha / 2}}
$$

Subsequently, the expectation can be approximated by

$$
\begin{gathered}
=\exp \left(-\pi \lambda r^{2} \sum_{n_{1}=1}^{N_{p}} \frac{w_{n_{1}}}{\sqrt{\pi}}\left(\frac{2}{\alpha} C^{\frac{2}{\alpha}} \frac{\pi}{\sin \left(\frac{\pi(\alpha-2)}{\alpha}\right)}\right.\right. \\
\left.\left.-\sum_{n_{G L}=1}^{N_{G L}} \frac{c_{i}}{2} \frac{C}{C+\left(\frac{x_{i}+1}{2}\right)^{\alpha / 2}}\right)\right) \\
=\exp \left(-\pi \lambda r^{2} f_{S F R}(T, n)\right)
\end{gathered}
$$

Substituting (17-20) into (7), we obtain the desired result.

\section{REFERENCES}

1. Dinh Thi Thai, M., et al. BER of QPSK using MRC reception in a composite fading environment. in 2012 International Symposium on Communications and Information Technologies (ISCIT). 2012.

2. Andrews, J.G., F. Baccelli, and R.K. Ganti. A new tractable model for cellular coverage. in, 2010 48th Annual Allerton Conference on Communication, Control, and Computing (Allerton). 2010.

3. Thomas David Novlan, R.K.G., Arunabha Ghosh, Jeffrey G. Andrews, Analytical Evaluation of Fractional Frequency Reuse for OFDMA Cellular Networks. IEEE Transactions on wireless communications, 2011. 10: p. 4294-4305.

4. He Zhuang, T.O., A model based on Poisson point process for downlink $K$ tiers fractional frequency reuse heterogeneous networks. Physical Communication, 2014. Volume 13, Part B(Special Issue on Heterogeneous and Small Cell Networks): p. 3-12.

5. Dhillon, H.S., et al., Modeling and Analysis of K-Tier Downlink Heterogeneous Cellular Networks., IEEE Journal on Selected Areas in Communications, 2012. 30(3): p. 550-560.

6. Di Renzo, M., A. Guidotti, and G.E. Corazza, Average Rate of Downlink Heterogeneous Cellular Networks over Generalized Fading Channels: A Stochastic Geometry Approach. IEEE Transactions on Communications, 2013. 61(7): p. 3050-3071.

7. Stegun, M.A.a.I.A., Handbook of Mathematical Functions with Formulas, Graphs, and Mathematical Tables. 9th ed. 1972: Dover Publications.

8. Keeler, H.P., B. Blaszczyszyn, and M.K. Karray. SINRbased $k$-coverage probability in cellular networks with arbitrary shadowing. in, 2013 IEEE International Symposium on Information Theory Proceedings (ISIT). 2013.

9. Xiaobin, Y. and A.O. Fapojuwo. Performance analysis of poisson cellular networks with lognormal shadowed Rayleigh fading. in 2014 IEEE International Conference on Communications (ICC). 2014.

10. Sung Nok Chiu, D.S., Wilfrid S. Kendall, Joseph Mecke, Stochastic Geometry and Its Applications. 3rd Edition ed. 2013: Wiley. 